\title{
Bogoliubov space of a Bose-Einstein condensate and quantum spacetime fluctuations
}

\author{
J. I. Rivas, ${ }^{\text {^ }}$ A. Camacho ${ }^{\dagger}$ and E. Gökli周 \\ Departamento de Física, Universidad Autónoma Metropolitana-Iztapalapa \\ Apartado Postal 55-534, C.P. 09340, México, D.F., México.
}

(Dated: June 4, 2022)

\begin{abstract}
In the present work we consider the role that metric fluctuations could have upon the properties of a Bose-Einstein condensate. In particular we consider the Bogoliubov space associated to it and show that there are, at least, two independent ways in which the average size of these metric fluctuations could be, experimentally, determined. Indeed, we prove that the pressure and the speed of sound of the ground state define an expression allowing us to determine the average size of these fluctuations. Afterwards, an interferometric experiment involving Bogoliubov excitations of the condensate and the pressure (or the speed of sound of the ground state) provides a second and independent way in which this average size could be determined, experimentally.
\end{abstract}

PACS numbers: $04.50+\mathrm{h}, 04.20 . \mathrm{Jb}, 11.25 . \mathrm{Mj}, 04.60 . \mathrm{Bc}$

\section{INTRODUCTION}

The mathematical and physical difficulties plaguing all theoretical models behind a quantum theory of gravity [1, 2] have spurred the so-called quantum gravity phenomenology, a topic that can be defined as the observational and experimental search for deviations from Einstein's General Relativity, or from quantum field theory. It embodies deformed versions of the dispersion relation 3, 4, or deviations from the $1 / r-$ potential and violations of the equivalence principle [5, 6. Of course, these aforementioned cases do not exhaust the extant possibilities. The use of cold atoms, either bosonic or fermionic, in this context is a point already considered [7, 8. In particular the possibility of constraining the energy-momentum relation resorting to cold atoms has already shown us that this kind of systems could open up new landscapes in the context of gravitational physics [9].

In the present work we consider the role that metric fluctuations could have upon the properties of a BoseEinstein condensate. In particular we address the issue of conformal metric fluctuations. Let us explain, briefly, the meaning of this last sentence. In this context the main idea corresponds to a Minkowskian background and in addition small spacetime fluctuations are also present, and they are a consequence of some quantum gravity scenario. One of the assumptions in this approach is related to the fact that these spacetime fluctuations emerge as classical fluctuations of the background metric. There are several possibilities around the type of fluctuations that can be considered [10]. among the huge spectrum of possibilities we may find the so-called conformal fluctuations 11, 12. They can be considered, mathematically, the simplest case and entail a redefinition of the inertial mass. In other words, the average size of this kind of fluctuations, say $\gamma$, appears in the corresponding motion

\footnotetext{
* jirs@xanum.uam.mx

$\dagger$ acq@xanum.uam.mx

$\ddagger$ cbi920000370@xanum.uam.mx
}

equations in the form $m /(1+\gamma)$, where $m$ denotes the usual inertial mass (when no fluctuations are present). Clearly, an experimental outcome detects $m /(1+\gamma)$, and not $m$ or $\gamma$ separately. Of course, a possible objection to the analysis of conformal fluctuations could be related to its simplicity, which in physical terms implies a redefinition of the inertial mass. In this work we will show that even the simplest case, i.e., conformal fluctuations, is endowed with a richness that leads us to detectable effects.

The idea in the present work is related to the possibility of, by means of two or more experimental proposals, deducing, separately, $m$ and $\gamma$. It will be proved that, the Bogoliubov space of a Bose-Einstein offers this option. Indeed, we consider the Bogoliubov space associated to a Bose-Einstein condensate and show that there are, at least, two independent ways in which the average size of these metric fluctuations can be, experimentally determined. Firstly, we consider the many-particle Hamiltonian of a bosonic gas, immersed in a homogeneous gravitational field, and, in addition, the effects of the metric fluctuations upon the inertial mass will be introduced. It will be shown that the pressure and speed of sound of the ground state of the Bogoliubov space of the condensate allow us to put forward and experiment which, in principle, determines the average size of these fluctuations. As a by product the value of $m$, i.e., the bare mass, can also be obtained. Secondly, we analyze an interferometric experiment, resorting to Bogoliubov excitations, and deduce the phase shift induced by the gravitational field and the metric fluctuations. It will be proved that this gravity-induced phase shift together with the pressure (or the speed of sound) of the ground state of the Bogoliubov space imply two expressions which determine the size of these metric fluctuations, and also of $m$. In order to understand better this argument, let us denote by $v_{s}$ the speed of sound of the ground state of the Bogoliubov space, and by $\Delta \phi$ the aforementioned gravity-induced phase shift of two Bogoliubov excitations. It will be shown that, roughly said, $m /(1+\gamma)=f_{1}\left(v_{s}, a, N, V, g\right)$, whereas $m / \sqrt{(1+\gamma)}=f_{2}(\Delta \phi, a, N, V, g)$. In this last 
two expression $f_{1}$ and $f_{2}$ are two functions (deduced in this work), and $N, V, a$, and $g$, denote the number of particles, volume of the container of the bosonic gas, scattering length, and, finally, acceleration of gravity, respectively. Clearly, $f_{2} / f_{1}=\sqrt{1+\gamma}$. In other words, the experimental deduction of $f_{1}$ and $f_{2}$ leads us to the determination of a characteristic of this metric fluctuations, namely, $\gamma=\left(f_{2} / f_{1}\right)^{2}-1$. In a similar way we may find an expression for $m$.

\section{METRIC FLUCTUATIONS AND A WEAKLY INTERACTING BOSE GAS}

\section{A. Metric fluctuations}

Now we, succinctly, address the issue of metric fluctuations. In this context a spacetime is present, the one can be regarded as a classical background on which classical fluctuations exist. [11. We suppose they are a consequence of a quantum theory of gravity where the microscopic structure of spacetime exhibits quantum fluctuations. These spacetime fluctuations modify our current physics. Indeed, they entail a change of the motion equations. In this general scheme these fluctuations imply a modified Schrödinger equation, in which the Laplacian operator becomes $\left(\delta^{i j}+\gamma^{i j}\right) \partial_{i} \partial_{j}$, where $\gamma^{i j}>0$. [11, 12]. The simplest case involves the so-called conformal fluctuations defined by the following conditions: $\gamma^{i j}=0$, if $i \neq j$, whereas $\gamma^{x x}=\gamma^{y y}=\gamma^{z z}=\gamma$. We may rephrase this last assertion stating that the idea of conformal fluctuations is depicted by matrices proportional to the unit matrix. Additionally, these fluctuations can be comprehended (at least partially) as redefinitions of the inertial mass [12]. The new inertial mass for an atom $\left(m^{e f f}\right)$ is now given by

$$
m^{e f f}=m(1+\gamma)^{-1}
$$

The parameter $\gamma$ encodes the information concerning the metric fluctuations and its value depends on the particle species under consideration. Clearly, they have to be considered very small (otherwise its existence would be a proved experimental fact). In addition, we do assume that it has a particular sign, namely, it has to be positive [12. Additionally, $(\gamma)$ depends upon the type of particle [11, 12].

This last expression contains an experimental hurdle. Indeed, notice that a kinematical experiment detects the relation $m(1+\gamma)^{-1}$, but not $m$ or $\gamma$, separately. At this point we pose the main question that will be addressed in this work: Could $m$ and $\gamma$ be measured separately?

\section{B. Weakly interacting gas}

As mentioned before, the main idea in this work involves an experimental proposal for the detection of the bare inertial mass, here denoted by $m$, and the average size of our conformal fluctuations, the parameter $\gamma$. Indeed, the Laplacian operator becomes, under this condition [11, 12].

$$
\Delta_{\gamma}=\left(\delta^{i j}+\gamma^{i j}\right) \partial_{i} \partial_{j}
$$

This modification will be considered in the $N$-body Hamiltonian operator (assuming that the gas is so dilute that only the two-body interaction potential is required [13]). Our model will be a Bose-Einstein gas enclosed in a container of volume $V$, particles of the gas are atoms with passive gravitational mass $m$ and located at a height $l$ with respect to the Earths surface. The interaction between two particles will be assumed to be dominated by $s$-scattering, i.e., the temperature of the system is very low $(k a<<1$, where $\vec{k}$ and $a$ are the wave vector and the scattering length, respectively) [14. This entails the following Hamiltonian for the $N$-body system.

$$
\begin{gathered}
\hat{H}=\sum_{\vec{k}=0} \frac{\hbar^{2} k^{2}}{2 m}(1+\gamma) \hat{a}_{\vec{k}}^{\dagger} \hat{a}_{\vec{k}} \\
+\frac{U_{0}}{2 V} \sum_{\vec{k}=0} \sum_{\vec{p}=0} \sum_{\vec{q}=0} \hat{a}_{\vec{p}}^{\dagger} \hat{a}_{\vec{q}}^{\dagger} \hat{a}_{\vec{p}+\vec{k}} \hat{a}_{\vec{q}-\vec{k}} \\
+\sum_{\vec{k}=0} m g l \hat{a}_{\vec{k}}^{\dagger} \hat{a}_{\vec{k}}, \\
U_{0}=\frac{4 \pi a \hbar^{2}}{m}(1+\gamma) .
\end{gathered}
$$

Demanding $\gamma=0$ we recover the usual result [15]. These operators $\left(\hat{a}_{\vec{k}}\right.$ and $\left.\hat{a}_{\vec{k}}^{\dagger}\right)$ are bosonic creation and annihilation operators, and fulfill the usual Bose commutator relations. Very close to the temperature $T=0$, the second term in this Hamiltonian becomes [15]

$$
\begin{array}{r}
\sum_{\vec{k}=0} \sum_{\vec{p}=0} \sum_{\vec{q}=0} \hat{a}_{\vec{p}}^{\dagger} \hat{a}_{\vec{q}}^{\dagger} \hat{a}_{\vec{p}+\vec{k}} \hat{a}_{\vec{q}-\vec{k}}=N^{2}+2 N \sum_{\vec{k} \neq 0} \hat{a}_{\vec{k}}^{\dagger} \hat{a}_{\vec{k}} \\
+N \sum_{\vec{k} \neq 0}\left(\hat{a}_{\vec{k}}^{\dagger} \hat{a}_{-\vec{k}}^{\dagger}+\hat{a}_{\vec{k}} \hat{a}_{-\vec{k}}\right) .
\end{array}
$$

With this approximation the $N$-body Hamiltonian has the following structure 


$$
\begin{array}{r}
\hat{H}=\frac{U_{0} N^{2}}{2 V}+m g l N+ \\
\sum_{\vec{k} \neq 0}\left[\frac{\hbar^{2} k^{2}}{2 m}(1+\gamma)+m g l+\frac{U_{0} N}{V}\right] \hat{a}_{\vec{k}}^{\dagger} \hat{a}_{\vec{k}} \\
+N \frac{U_{0}}{2 V}\left[\hat{a}_{\vec{k}}^{\dagger} \hat{a}_{-\vec{k}}^{\dagger}+\hat{a}_{\vec{k}} \hat{a}_{-\vec{k}}\right] .
\end{array}
$$

This Hamiltonian can be diagonalized introducing the Bogoliubov transformations [14]

$$
\begin{aligned}
& \hat{b}_{\vec{k}}=\frac{1}{\sqrt{1-\alpha_{k}^{2}}}\left[\hat{a}_{\vec{k}}+\alpha_{k} \hat{a}_{-\vec{k}}^{\dagger}\right], \\
& \hat{b}_{\vec{k}}^{\dagger}=\frac{1}{\sqrt{1-\alpha_{k}^{2}}}\left[\hat{a}_{\vec{k}}^{\dagger}+\alpha_{k} \hat{a}_{-\vec{k}}\right] .
\end{aligned}
$$

In this last expression the following definitions have been introduced

$$
\begin{gathered}
\epsilon_{k}=\frac{\hbar^{2} k^{2}}{2 m}(1+\gamma)+m g l \\
\alpha_{k}=1+\frac{V \epsilon_{k}}{U_{0} N}-\sqrt{\frac{V \epsilon_{k}}{U_{0} N}} \sqrt{2+\frac{V \epsilon_{k}}{U_{0} N}} .
\end{gathered}
$$

They fulfill the same algebra related to $\hat{a}_{\vec{k}}$ and $\hat{a}_{\vec{k}}^{\dagger}$, i.e., they are also bosonic operators. The final form for our Hamiltonian is

$$
\begin{array}{r}
\hat{H}=\frac{U_{0} N^{2}}{2 V}+m g l N \\
+\sum_{\vec{k} \neq 0}\left\{\sqrt{\epsilon_{k}\left(\epsilon_{k}+\frac{2 U_{0} N}{V}\right)} \hat{b}_{\vec{k}}^{\dagger} \hat{b}_{\vec{k}}\right. \\
\left.-\frac{1}{2}\left[\frac{U_{0} N}{V}+\epsilon_{k}-\sqrt{\epsilon_{k}\left(\epsilon_{k}+\frac{2 U_{0} N}{V}\right)}\right]\right\} .
\end{array}
$$

The last summation diverges, a result already known [16. 17], and this divergence disappears introducing the so-called pseudo-potential method, which implies that we must perform the following substitution [17]

$$
\begin{gathered}
-\frac{1}{2}\left[\frac{U_{0} N}{V}+\epsilon_{k}-\sqrt{\epsilon_{k}\left(\epsilon_{k}+\frac{2 U_{0} N}{V}\right)}\right] \\
-\frac{1}{2}\left[\frac{U_{0} N}{V}+\epsilon_{k}-\sqrt{\epsilon_{k}\left(\epsilon_{k}+\frac{2 U_{0} N}{V}\right)}-\frac{1}{2 \epsilon_{k}}\left(\frac{U_{0} N}{V}\right)^{2}\right] .
\end{gathered}
$$

Finally, this last summation will be approximated by an integral. It is noteworthy to mention that the original expression has as lower limit the condition $k \neq 0$, which implies that the integral has as lower limit the value $m g l$. In other words,

$$
\begin{aligned}
& -\frac{1}{2} \sum_{\vec{k} \neq 0}\left[\frac{U_{0} N}{V}+\epsilon_{k}-\sqrt{\epsilon_{k}\left(\epsilon_{k}+\frac{2 U_{0} N}{V}\right)}-\frac{1}{2 \epsilon_{k}}\left(\frac{U_{0} N}{V}\right)^{2}\right] \\
& =-\frac{\hbar^{2} V}{8 m \pi^{2}}\left(\frac{8 \pi a N}{V}\right)^{5 / 2} \int_{m g l}^{\infty} f(x) d x .
\end{aligned}
$$

In this last expression we have that

$$
f(x)=x^{2}\left[1+x^{2}-x \sqrt{2+x^{2}}-\frac{1}{2 x^{2}}\right] .
$$

In this last expression we have that

$$
x=\sqrt{\frac{\epsilon_{k} V}{U_{0} N}} .
$$

With these conditions we deduce the final structure of the $N$-body Hamiltonian

$$
\hat{H}=E_{0}+\sum_{\vec{k} \neq 0} E_{k} \hat{b}_{\vec{k}}^{\dagger} \hat{b}_{\vec{k}} .
$$

In this last expression $E_{0}$ denotes the energy of the ground state of the corresponding Bogoliubov space [14].

$$
\begin{gathered}
E_{0}=\frac{2 \pi a \hbar^{2} N^{2}(1+\gamma)}{m V}\left[1+\frac{128}{15} \sqrt{\frac{a^{3} N}{V \pi}}\right. \\
\left.\left(1-\frac{15}{16 \sqrt{2}} \sqrt{\frac{m^{2} g l V}{4 \pi a \hbar^{2} N(1+\gamma)}}\right)\right]+N m g l .
\end{gathered}
$$

On the other hand, we have that the energy of the Bogoliubov excitations $\left(E_{k}\right)$ is given by 14

$$
E_{k}=\sqrt{\epsilon_{k}\left(\epsilon_{k}+\frac{2 U_{0} N}{V}\right)} .
$$

Concerning 16 , if we impose the condition of vanishing gravitational constant, i.e. $g=0$, then we recover the usual Hamiltonian [17.

\section{Speed of sound and pressure of the ground state}

The pressure $\left(P_{0}=-\frac{\partial E_{0}}{\partial V}\right)$ and speed of sound $\left(v_{s}=\right.$ $\left.\sqrt{-\frac{V^{2}}{N m} \frac{\partial P_{0}}{\partial V}}\right)$ associated to the ground state of the Bogoliubov space become, respectively

$$
\begin{array}{r}
P_{0}=\frac{2 \pi a \hbar^{2} N^{2}(1+\gamma)}{m V^{2}}\left[1+\frac{192}{15} \sqrt{\frac{a^{3} N}{V \pi}}\right. \\
\left.\left(1-\frac{5}{8 \sqrt{2}} \sqrt{\frac{m^{2} g l V}{4 \pi a \hbar^{2} N(1+\gamma)}}\right)\right],
\end{array}
$$




$$
\begin{array}{r}
v_{s}^{2}=\frac{4 \pi a \hbar^{2} N(1+\gamma)^{2}}{m^{2} V}\left[1+16 \sqrt{\frac{a^{3} N}{V \pi}}\right. \\
\left.\left(1-\frac{1}{2 \sqrt{2}} \sqrt{\frac{m^{2} g l V}{4 \pi a \hbar^{2} N(1+\gamma)}}\right)\right] .
\end{array}
$$

A fleeting glimpse at these last expressions tells us that they depend upon the bare inertial mass and on the size of the fluctuations, i.e, upon $m$ and $\gamma$, respectively.

Notice that they imply the following relation

$$
\begin{gathered}
\frac{m}{\sqrt{1+\gamma}}=\frac{v_{s}^{2} \pi a \hbar^{2} N^{3}(1+\alpha)^{2}-P_{0}^{2} V^{3}\left(1+\frac{5}{4} \alpha\right)}{2 \alpha v_{s}^{2} \pi a \hbar^{2} \beta(1+\alpha)-P_{0}^{2} V^{3} \alpha \beta}, \\
\alpha=\frac{192}{15} \sqrt{\frac{a^{3} N}{V \pi}} ; \quad \beta=\frac{5}{8 \sqrt{2}} \sqrt{\frac{g l V}{4 \pi a \hbar^{2} N}} .
\end{gathered}
$$

Clearly, it does not contain the usual relation, namely, $m(1+\gamma)^{-1}$. Consider the kinematical relation associated to $U_{0}$ (see (4)). If $F$ denotes the right-hand side of (21), then we obtain

$$
\begin{gathered}
\sqrt{(1+\gamma)}=\frac{F U_{0}}{4 \pi a \hbar^{2}}, \\
m=\frac{F^{2} U_{0}}{4 \pi a \hbar^{2}} .
\end{gathered}
$$

These two last expression allows us to deduce $m$ and $\gamma$, separately. The right-hand side of them involves parameters which can be detected experimentally.

\section{Bogoliubov excitations and metric fluctuations}

We now present a second manner in which $m$ and $\gamma$ could be detected separately. Here we will resort to the properties of Bogoliubov excitations of the condensate. It is already known [14 that, even at $T=0$, the presence of two-body interactions entail the existence of excitations in the condensate [15, 17, whose energy is given by (18). At this point we consider two Bogoliubov excitations located, initially, at point (A) (see figure 1), and whose wave vector fulfills the following condition

$$
\frac{\hbar^{2} k^{2}}{2 m}(1+\gamma)+m g l>\frac{2 U_{0} N}{V}
$$

Then, we have, approximately

$$
E_{k}=\frac{\hbar^{2} k^{2}}{2 m}(1+\gamma)+m g l+\frac{4 \pi a \hbar^{2} N(1+\gamma)}{m V}
$$

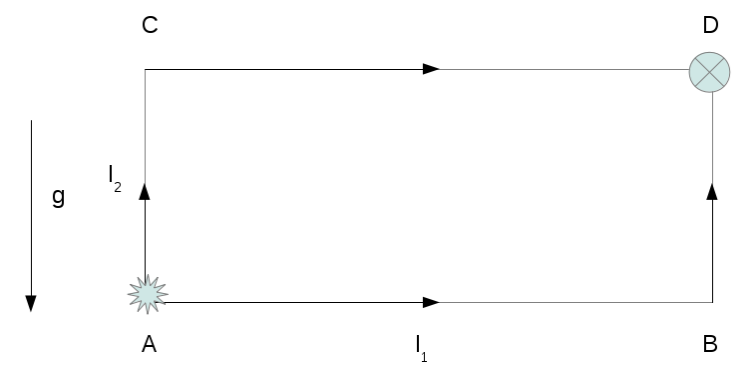

FIG. 1. The interferometer with arm lengths $l_{1}$ and $l_{2}$. The source for the Bose-Einstein-Condensates is located at point A, where it is coherently split into two sub-condensates which travel along different paths. Finally, due to the different times of flight, the phase shift $\Delta \phi$ can be detected at the detector at point $\mathrm{D}$ by means of the interference pattern.

These conditions allow us to consider an interferometric proposal along the lines of a semi-classical approximation. In other words, we consider an experiment similar to the Colella-Overhauser-Werner idea [18 21]. This last experiment shows the effects of the gravitational field of the Earth upon the phase shift of two neutron beams. Here we consider the same kind of proposal but with a different intention. Indeed, we seek for an extra expression whose dependence is not of the kind $\left[m(1+\gamma)^{-1}\right]^{s}$, where $s$ is a real number. We now resort to the WKB approximation in order to deduce the corresponding gravityinduced phase shift [22].

The time of flight for the beam moving along the path (A) $-(\mathrm{B})-(\mathrm{D})$ reads

$$
\begin{aligned}
{ }^{(1)} t= & \frac{m l_{1}}{(1+\gamma) \hbar k_{0}}+\frac{1}{g}\left[\frac{(1+\gamma)}{m} \hbar k_{0}\right. \\
& \left.-\sqrt{\left(\frac{(1+\gamma)}{m} \hbar k_{0}\right)^{2}-2 g l_{2}}\right]
\end{aligned}
$$

Concerning (A)-(C)-(D) the time of flight is

$$
\begin{aligned}
& { }^{(2)} t=\frac{1}{g}\left[\frac{(1+\gamma)}{m} \hbar k_{0}-\sqrt{\left(\frac{(1+\gamma)}{m} \hbar k_{0}\right)^{2}-2 g l_{2}}\right] \\
& +\sqrt{\frac{l_{1}^{2}}{\left(\frac{(1+\gamma) \hbar k_{0}}{m}\right)^{2}-2 g l_{2}}} .
\end{aligned}
$$

In these last expressions $k_{0}$ denotes the wave number at point (A). These expressions are valid for short times of flight or for large velocities for the motion of the centre of mass of the BEC. Additionally, we have also assumed that the line passing through points (B) and (D) is parallel to the direction of the gravitational field. If this line forms an angle $\theta$ with the gravitational field, then our expression remains valid but now we have an effective gravitational acceleration given by $g \cos (\theta)$. Clearly, with these two flight times we may deduce, easily, the 
difference in time of arrival 22 and, in consequence, the gravity-induced phase shift, here denoted by $\Delta \phi$.

$$
\Delta \phi=\frac{m^{2} g l_{1} l_{2}}{(1+\gamma) \hbar^{2} k_{0}}\left[1-\frac{2 m^{2} g l_{2}}{(1+\gamma) \hbar^{2} k_{0}^{2}}\right] .
$$

Introducing the condition $\gamma=0$ we recover the COW result [22]. We now notice that this phase shift contains a dependence of the kind $m^{2} /(1+\gamma)$. Taking the predominant term in the expression for the gravity-induced phase shift and resorting to our previous results concerning the speed of sound of the ground state (see (20p) we may, separately, deduce $m$ and $\gamma$. Indeed (here $\lambda_{0}$ denotes the wavelength at (A) divided by $2 \pi$ ),

$$
m=\frac{v_{s} \Delta \phi \hbar^{2}}{g l_{1} l_{2} \lambda_{0}} \sqrt{\frac{V}{4 \pi a N}}\left[1-16 \sqrt{\frac{a^{3} N}{\pi V}}\right] .
$$

In a similar way we find that

$$
\gamma=\frac{v_{s}^{2} \Delta \phi \hbar^{2}}{g l_{1} l_{2} \lambda_{0}} \frac{V}{4 \pi a N}\left[1-32 \sqrt{\frac{a^{3} N}{\pi V}}\right]-1 .
$$

In the deduction of these last two expressions we have resorted to the speed of sound of the ground state, nevertheless, the pressure could have been used, as well.

\section{CONCLUSIONS}

We have introduced into the $N$-body Hamiltonian the effects of conformal fluctuations of the metric, which are usually associated to a redefinition of the inertial mass of the involved particles, i.e., mass appears as $m(1+\gamma)^{-1}$. Two different manners in which, experimentally, these two parameters could be detected have been put forward.

At this point we must emphasize the fact that the present work contains an experimental proposal aimed at the detection of some properties of quantum fluctuations. Firstly, the deduction of the pressure and speed of sound of the ground state of the Bogoliubov space have been calculated. These expressions allow us, when compared against $U_{0}$, to achieve our goal. Secondly, an interferometric proposal, resorting to Bogoliubov excitations has been analyzed. It has been shown that the gravityinduced phase shift, along with the pressure (or speed of sound) of the ground state, renders a second way in which we may, in an experiment, deduce, separately, $m$ and $\gamma$. It is noteworthy to mention that this approach can be used in the context of deformed dispersion relations. Let us explain better this idea. The breakdown of Lorentz symmetry appears as a consequence in some quantum gravity models 23 . The possibilities that cold atoms offer in this direction has already been considered, though the role that Bogoliubov excitations could play in this context has not been analyzed yet. In other words, the present proposal can be considered as some kind of complementary study to 9 .

Of course, an important point in this context is the feasibility of the detection of the pressure and speed of sound of the ground state of a Bose-Einstein condensate. The speed of sound of a Bose-Einstein condensate, comprising sodium atoms, has already been measured [24, though, of course, more experimental work is needed in this realm. In other words, the present proposal does not seem to be, in this direction, very far from the present technology. As an additional bonus related to the present work let us mention the connection with the Einstein Equivalence Principle (EEP) [25], namely, the famous "semicolon goes to coma rule". This principle tells us that locally the laws of physics are the special-relativistic laws. We may rephrase this statement asserting that locally the gravitational field can be gauged away. In other words, in a freely falling frame the pressure and speed of sound, related to the ground state of the Bogoliubov space must be given by our expression, if $g=0$. Similarly, the gravity-induced phase shift shall vanish. This kind of experiments are, currently, a hot topic in gravitational physics [26, since nowadays it is possible to create condensates, in a regular basis, under microgravity. The present idea can also be considered as a proposal for the use of Bogoliubov fluctuations as an additional tool for experiments in fundamental physics. This kind of experiments are, currently, a hot topic in gravitational physics [26], since nowadays it is possible to create condensates, in a regular basis, under microgravity. The present idea can also be considered as a proposal for the use of Bogoliubov fluctuations as an additional tool for experiments in fundamental physics.

Finally, a heated debate concerning the role that atom interferometry plays in the context of gravitational shift (universality of clock rates) erupted recently [27, 28. Clearly, at least one of these two interpretations has to be wrong. The aforementioned discussion shall not be neglected since it addresses an important and fundamental aspect in gravitational physics. The present proposal puts forward the possibility of carrying out, with Bogoliubov excitations, a similar experiment. In other words, it offers the option of an interferometric experiment without resorting to atoms [27, 28], or to neutrons [7, 8]. Maybe this new case could shed some light upon this debate.

\section{ACKNOWLEDGMENTS}

JIRS acknowledges CONACyT grant No. 18176. E. G. acknowledges UAM-I for the grant received. 
[1] C. N. Kozameh and M. F. Parisi, Class. Quantum Grav. 21, 2617 (2004).

[2] K. Becker, M. Becker, and J. H. Schwarz. String Theory and M-Theory: A Modern Introduction (Cambridge University Press 2007).

[3] G. Amelino-Camelia, N. Loret, G. Mandanici, F. Mercati, Int. J. Mod. Phys. D 19, 2385 (2010).

[4] G. Amelino-Camelia, Int. J. Mod. Phys. D 11, 35 (2002).

[5] G. Amelino-Camelia, C. Lämmerzahl, A. Macías, and H. Müller, in: Gravitation and Cosmology: 2nd Mexican Meeting on Mathematical and Experimental Physics, 2005, edited by A. Macías, C. Lämmerzahl, and D. Núñez (American Institut of Physics 2005) p. 30.

[6] E. Fischbach and C. L. Talmadge, The Search for NonNewtonian Gravity (Springer-Verlag, New York, 1999).

[7] D. Colladay and P. McDonald, Phys. Rev. D70, 125007 (2004).

[8] D. Colladay and P. McDonald, Phys. Rev. D73, 105006 (2006).

[9] G. Amelino-Camelia et al, Phys. Rev. Lett. 103: 171302 (2009).

[10] L. B. Lawrence, Quantum Fluctuations of Spacetime (World Scientific Publishing Co 2005).

[11] E. Göklü, C. Lämmerzahl, Class. Quantum Grav. 25, 105012 (2008).

[12] E. Göklü, C. Lämmerzahl, A. Camacho, and A. Macías, Class. Quantum Grav. 26, 225010 (2009).

[13] R. K. Pathria, Statistical Mechanics (ButterworthHeinemann, Oxford, 1996).

[14] L. Pitaevski and S. Stringari, Bose-Einstein Condensa- tion (Clarendon Press, Oxford, 2003).

[15] C. J. Pethick and H. Smith, Bose-Einstein Condensation in Dilute Gases (Cambridge University Press, Cambridge, 2004).

[16] G. F. Gribakin and V. V. Flambaum, Phys. Rev. A48, 546-563 (1993).

[17] M. Ueda, Fundamentals and New Frontiers of BoseEinstein Condensation (World Scientic, Singapore, 2010).

[18] A. W. Overhauser and R. Colella, Phys. Rev. Lett. 33, 11237-1239 (1074).

[19] J. L. Straudemann et al, Phys. Rev. A21, 1419-1438 (1980).

[20] D. M. Greenberg and A. W. Overhauser, Rev. Mod. Phys. 51, 43-78 (1979).

[21] K. C. Littrell, B. E. Allman, and S. A. Werner, Phys. Rev. A56, 1767-1780 (1997).

[22] J. J. Sakurai, Modern Quantum Mechanics (AddisonWesley Publishing Company, New York, 1994).

[23] G. Amelino-Camelia and L. Smolin, Physical Review D80, 084017 (2009).

[24] M. R. Andrews et al, Phys. Rev. Lett. 70, 553-556 (1997).

[25] C. M. Will Theory and Experiment in Gravitational Physics, (Cambridge University Press, Cambridge, 1993).

[26] C. van Zoest et al, Science 328, 1540-1543 (2010).

[27] A. Peters et al, Nature 463, 926-929 (2010).

[28] P. Wolf et al, Class. Quant. Grav. 28, 145017 (2011). 